\title{
Über das Problem der Nachbargebiete im Raum.
}

\section{Von Heinrich Tietze in Wien.}

In einem unlängst erschienenen Aufsatz von $P$. Wernick e ${ }^{1}$, , der sich mit dem bekannten Vierfarbenproblem beschäftigt, wird die Frage nach der sogenannten chromatischen Zahl des (dreidimensionalen) Raumes aufgeworfen. $\mathrm{Zu}$ dieser $\mathrm{Zahl}$ führt die Aufgabe, bei einer vorgelegten Einteilung des Raumes (oder eines Raumteiles) den einzelnen Teilen Farben zuzuweisen, derart, daß 1. Teile des Raumes, die eine Begrenzungsfläche gemein haben, verschiedene Farben erhalten, 2. eine möglichst geringe Zahl von Farben zur Verwendung kommt. Unter der chromatischen Zahl des Raumes versteht man dann die geringste Zahl von Farben, mit der man bei einer beliebigen Raumeinteilung auslangt. Daß nun die chromatische Zahl des dreidimensionalen Raumes - und dann natürlich auch jedes Raumes von höherer Dimension - unendlich groß ist, hat bereits Stäckel ${ }^{2}$ ) gezeigt, indem er ein Verfahren angab, im dreidimensionalen Raum eine beliebig vorgegebene Anzahl von Nachbargebieten zu konstruieren. Dabei versteht man unter $n$ "Nachbargebieten" $n$ Gebiete derart, dalo jedes mit jedem eine gemeinsame Begrenzungsfläche aufweist. ${ }^{3}$ )

Stäckel weist nun darauf hin, daß die Nachbargebiete, die er konstruiert, nicht ïberall konvex sind und stellt die Frage, ob die

1) Math. Ann. 58, 2) Zeitschrift f. Math. u. Phys., 42. Jahrgang. Ein älterer Beweis hiefür von Frederick Guthrie, Proc. Roy. Soc. Edinburgh X. Ein dritter Beweis wurde neverdings von $\mathrm{Di}$ $\mathrm{x}$ o $\mathrm{n}$ gegeben, Messenger 32. ${ }^{3}$ ) Ein besonders einfaches Beispiel von $n$ Nachbargebieten erhält man folgendermaßen: Man lege eine quadratische Platte auf die $x y$-Ebene, derart, dab die Quadratseiten der $x$ - bezw. der $y$-Achse parallel werden, und auf diese quadratische

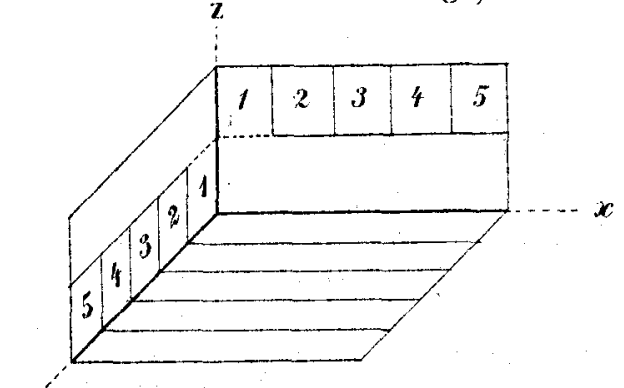

Fig. 1. Platte eine zweite kongruente. Hierauf werde die eine Platte durch Schnitte parallel zur $x z$-Ebene, die andere durch Schnitte parallel der $y z$-Ebene je in $n$ Stăbe geteilt, und der $m$ te Stab der einen Platte mit dem $m$ ten Stab der zweiten Platte $(m=1,2, \ldots n)$ zu einem Körper verschmolzen (s. Fig. 1. für den Fall $n=5$ ). 
Anzahl der konvexen Nachbargebiete im dreidimensonalen Raum begrenzt sei. ${ }^{1}$ ) In den folgenden Zeilen soll nun der Beweis geführt werden, daß diese Frage zu verneinen ist, indem gezeigt wird, daf man eine beliebig vorgegebene Anzahl von Nachbargebieten herstellen kann, die sämtlich konvexe Polyeder sind.

Ehe wir an diesen allgemeinen Beweis herantreten, soll ein Beispiel dafür betrachtet werden, daß man sechs konvexe Polyeder konstruieren kann, die ein System von Nachbargebieten bilden. Daf man fünf derartige Polyeder angeben kann, bemerkt schon Stäckel. Er führt hiefür als Beispiel die Zerlegung eines Tetraeders $A B C D$ in ein Mitteltetraeder $\operatorname{EFGH}$ und vier dreiseitige Pyramidenstumpfe $A B C E F G, A B D E F H, A C D E G H, B C D F G H$ an (s. Fig. 2). Dabei sollen der Einfachheit halber die Tetraeder $A B C D$ und $E F G H$ regulär und mit paarweise parallelen Kanten angenommen werden und es sollen ihre Mittelpunkte zusammen fallen.

Aus der besprochenen Zerlegung von $A B C D$ wollen wir nun eine andere ableiten, bei der das Tetraeder in 6 konvexe Nachbargebiete zerfällt. Hiezu wähle man auf den Strecken $B F$ und $F H$

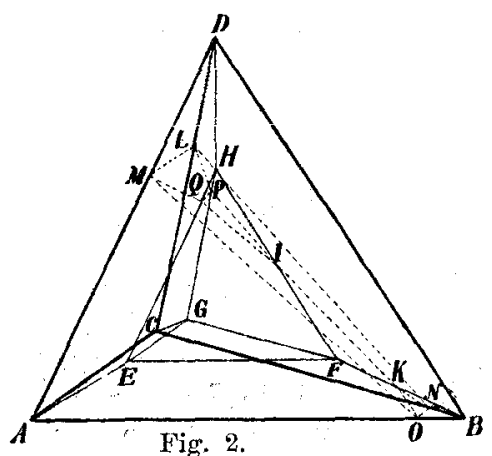

Fig. 2.

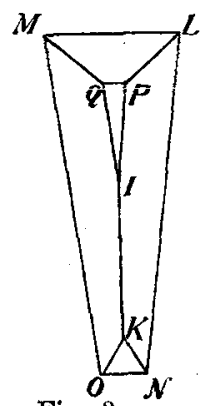

Fig. 3.

bezw. die Punkte $K, I$ und lege durch dieselben eine zu $A C$ parallele Ebene. Den Durchsehnitt dieser Ebene mit dem Tetraeder $A B C D$ zeigt Fig. 3.

Läßt man nun in dem Tetraederteil ACLMNO die ursprüngliche Einteilung bestehen, während man den Tetraederteil $B D L M N O$ als ein einziges Teilpolyeder auffaßt, so erhält man eine Einteilung von $A B C D$ in die 6 konvexen Polyeder EFGIPQ, $A C N O K E F G, A M Q I K O E F, A O L M E G P Q, C L P I K N F G$, $B D L M N O$, die ein System von Nachbargebieten darstellen.

Das eben entwickelte Beispiel, welches zeigt, daß man mehr als fünf konvexe Nachbargebiete konstruieren kann, legt die Vermutung nahe, daß die Zahl der konvexen Nachbargebiete beliebig.

1) Übrigens findet sich diese Fragestellung schon bei Frederick Guthrie angedeutet (a. a. $\Theta$.). 
groß werden kann. Diese Vermutung soll nun durch den Schluß von $n$ auf $n+1$ ibre Bestätigung finden.

Die konvexen Polyeder, mit denen wir dabei operieren, definieren wir in folgender Weise:

Es sei eine endliche Anzahl von Ebenen $E_{1}, E_{2}, \ldots$ gegeben und von jeder Ebene $E_{\boldsymbol{i}}$ sei die eine Seite als positiv, die andere als negativ bezeichnet. Gibt es dann einen Teil des Raumes, dessen Punkte in Bezug auf alle Ebenen $E_{i}$ auf der positiven Seite liegen, so nennen wir ihn ein Polyeder.

Es kann nun der Fall eintreten, daß wir einige der Ebenen $E_{i}$ fortlassen können, ohne daß sich das Polyeder dabei ändert (vergrößert). Es sind das diejenigen Ebenen $E_{i}$, die keine Punkte enthalten, die auf den positiven Seiten sämtlicher übrigen Ebenen liegen. Von diesen für die Definition des vorgelegten Polyeders unwesentlichen Ebenen wollen wir absehen. In Bezug auf die übrigen, für die Definition des Polyeders wesentlichen Ebenen $E_{i}$ definieren wir die Begrenzungspolygone wie folgt: Die Gesamtheit der Punkte einer Ebene $E_{i}$, die auf den positiven Seiten aller übrigen Ebenen liegen, heiße ein Begrenzungspolygon des Polyeders. Ein Polyeder mit $m$ Begrenzungspolygonen heiße ein $m$-flach ( $m$-eder).

In der gewählten Definition der Polyeder ist schon die Beschränkung auf konvexe Polyeder enthalten. Jedoch können sich bei unserer Definition die Polyeder auch ins Unendliche erstrecken, in welchem Fall sie als unendliche Polyeder bezeichnet werden sollen.

Wir betrachten nun Einteilungen des gesamten Raumes in unendliche Polyeder, die ein System von Nachbargebieten bilden. Aus einer solchen Einteilung in unendliche Polyeder läßt sich dann ein System (konvexer) im Endlichen gelegener Nachbargebiete erhalten, indem man jedes unendliche Polyeder in genügend großer Entfernung durch ebene Flächen begrenzt.

Wir nehmen an, wir hätten eine Einteilung $\mathfrak{I}_{n}$ des Raumes in $n$ unendliche Polyeder, wobei folgende Bedingungen erfüllt seien :

1. Je zwei Polyeder stoßen mit Teilen ihrer Begrenzungsflächen aneinander.

2. Eines der Polyeder ist ein 1-flach (Halbraum). Seine Begrenzungsebene werde mit $D$ bezeichnet. Von den beiden Halbräumen $d_{1}, d_{2}$, in die der gesamte Raum durch die Ebene $D$ zerfällt, bildet der eine, etwa $d_{1}$, das eben genannte 1-flach, während $d_{2}$ in die $n-1$ übrigen Polyeder zerfällt.

3. Es läßt sich eine Ebene $E$ von folgender Art legen.

a) Die Ebene $E$ schneidet jedes der $n$ Polyeder in einer (endlichen oder unendlichen) polygonalen Fläche. Dabei sollen diese Schnittpolygone im Innern der Polyeder liegen, also nicht etwa Begrenzungspolygone sein.

Aus a) folgt, daß $E$ nicht zu $D$ parallel sein kann. $E$ und $D$ werden sich also in einer Geraden $g$ schneiden. Durch $g$ wird $E$ in zwei Halbebenen $E_{1}, E_{2}$ zerlegt, von denen $E_{1}$ im Halbraum $d_{1}$, 
$E_{2}$ im Halbraum $d_{2}$ liegen möge. Durch die Elbene $E$ wird der gesamte Raum in zwei Halbräume geteilt, die mit $e_{1}, e_{2}$ bezeichnet werden sollen. Die in $e_{1}$, bezw. $e_{2}$ liegende Halbebene von $D$ werde mit $D_{1}$, bezw. $D_{2}$ bezeichnet.

b) Einer der Halbräume, die durch $E$ gebildet werden, etwa $e_{1}$, hat die Eigenschaft, daß in ihm alle $n$ Polyeder zu je zweien in Flächenstücken aneinandergrenzen. Wir könnten also den Halbraum $e_{2}$ fortlassen und die übrigbleibenden Teile der $n$ Polyeder würden wieder $n$ Nachbargebiete sein.

Bezüglich der Schnittfigur der Begrenzungsflächen der $n$ Polyeder mit der Ebene $E$ wissen wir zunächst: Durch den Strahl $g$ wird $E$ (s. Fig. 4 ) in zwei Halbebenen $E_{1}, E_{2}$ geteilt. $E_{1}$ stellt die Schnittfläche von $E$ mit dem 1-flach $d_{1}$ vor ; $E_{2}$ enthält die $n-1$

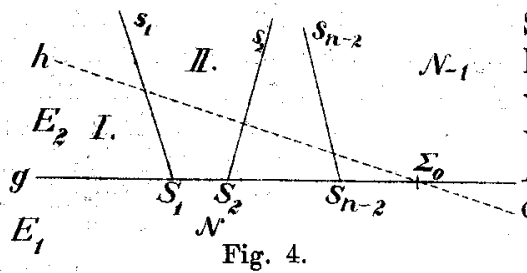

übrigen Schnittflächen. Sämtliche Schnittlinien (Strahlen, Halbstrahlen oder Strecken) außer $g$, die wir mit $s_{1}, s_{2}, \ldots$ bezeichnen wollen, liegen in der Halbebene $E_{2}$. Es sollen nun !noch die Be-

Fig. 4.

angen erfüllt sein :

c) Alle Schnittlinien $s$ schneiden $g$, u. zw. in durchaus verschiedenen Punkten $S_{1} S_{2}, \ldots$ Die Gerade $g$ wird dadurch in die Stücke $\infty S_{1}, S_{1} S_{2}, S_{2} S_{3}, \ldots$ zerlegt.

d) Jede der $(n-1)$ von den Polyedern gebildeten Schnittlächen, die die Halbebene $E_{2}$ erfüllen, soll in einem der eben genannten Stücke $\infty S_{1}, S_{1} S_{2}, \ldots$ an die Gerade $g$ grenzen. Da die Schnittflächen konvexe polygonale Flächen sind, so können sie nicht in mehr als einem Stück an $g$ grenzen.

Somit gibt es genau $n-2$ Schnittlinien s. Die Schnittflächen der Polyeder mit $E$ sind in Fig. 4 der Reihe nach mit I, II,.. $N-1, N$ bezeichnet.

Wir sehen nun für einen Augenblick von der Ebene $E$ ab und betrachten die ursprüngliche Teilung $\mathfrak{T}_{n}$ des Raumes in $n$ Polyeder. Insbesondere betrachten wir die Ebene $D$ und das System $\overleftarrow{\varsigma}$ von Schnittlinien, das auf $D$ von den übrigen Begrenzungsflächen ausgeschnitten wird. Dabei interessieren uns vorzüglich diejenigen dieser Schnittlinien, welche die Gerade $g$ schneiden. Die dadurch auf $g$ erhaltenen Schnittpunkte können aber offenbar keine andern als die Punkte $S_{1}, S_{2}, \ldots S_{n-2}$ sein und in jedem dieser Punkte kann nur eine dieser Schnittlinien die Gerade $g$ schneiden. Es mögen diese Schnittlinien der Reihe nach mit $t_{1}, t_{2}, \ldots t_{n-2}$ bezeichnet werden. Fassen wir speziell die Halbebene $D_{1}$ ins Auge, so wird dieselbe ein Bild wie Fig. 5 ergeben.

In den Punkten 1, 2, 3a) bis d) haben wir jene Voraussetzungen entwickelt und jene Bezeichnungen eingeführt, die es uns ermöglichen, folgenden Satz auszusprechen: 
Wenn wir unsere Teilung $\mathfrak{I}_{n}$ des Raumes in $n$ Polyeder dahin abändern, daß wir die Ebene $E$ als neue Begrenzungsfläche einführen, a. zw. so, daf im Halbraum $e_{1}$ die ursprünglichen Begrenzungstächen erhalten bleiben, hingegen im Halbraum $e_{2}$ alle ursprünglichen Begrenzungsfächen getilgt werden, somit $e_{2}$ als 1- Hach neu eingeführt wird, dann erhalten wir eine Teilung $\mathfrak{I}_{n+1}$ des Raumes in $n+1$ Polyeder, die ebenso wie die Teilung $\mathfrak{I}_{n}$ die Bedingungen 1 , $2 ., 3$ a), b), c), d) erfüllt.

Beweis: Auf Grund der Voraussetzung $3 \mathrm{~b}$ ) stoßen die $n$ in $e_{1}$ liegenden Reste der ur-

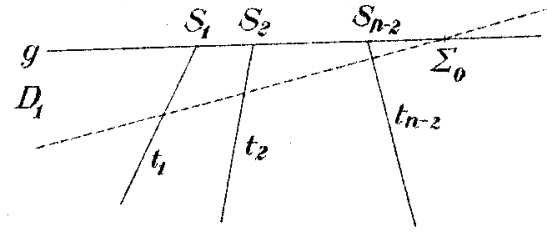

Fig. 5. sprünglichen $n$ Polyeder zu je zweien in Flächenstücken aneinander. Ferner grenzt auch das $(n+1)^{\text {te }}$ Polyeder, nämlich das 1 -flach $e_{z}$ wegen 3 a) an alle $n$ vorhergenannten Polyeder unserer neuen Einteilung $\mathfrak{I}_{n+1}$ Somit ist für dieselbe 1. erfüllt.

Desgleichen ist 2 . erfüllt, wobei an Stelle von $D, d_{1}, d_{2}$ die Gebilde $\vec{E}, e_{2}, e_{1}$ zu setzen sind.

Ferner wollen wir eine Ebene $F$ konstruieren und von derselben dann nachweisen, daß sie in Bezug auf die Teilung $\mathfrak{T}_{n+1}$ den Bedingungen entspricht, die in 3 . von der Ebene $E$ in Bezug auf die Teilung $\mathfrak{I}_{n}$ gefordert wurden.

Hiezu konstruieren wir zunächst in der Ebene $E$ eine Gerade $h$ derart, daß $h$ die sämtlichen Geraden $g, s_{1}, s_{2}, \ldots s_{n-2}$ im Endlichen in lauter verschiedenen Punkten $\Sigma_{0}, \Sigma_{1}, \Sigma_{2}, \ldots \Sigma_{n-2}$ schneidet, da 0 ferner alle Punkte $\Sigma_{1}, \Sigma_{2}, \ldots \Sigma_{n-2}$ in der Halbebene $E_{2}$ liegen, und dab auf jeder Geraden $s_{i}(i=1,2, \ldots n-2)$ zwischen $S_{i}$ und $\Sigma_{i}$ kein Schnittpunk von $s_{i}$ mit irgend einer anderen Geraden

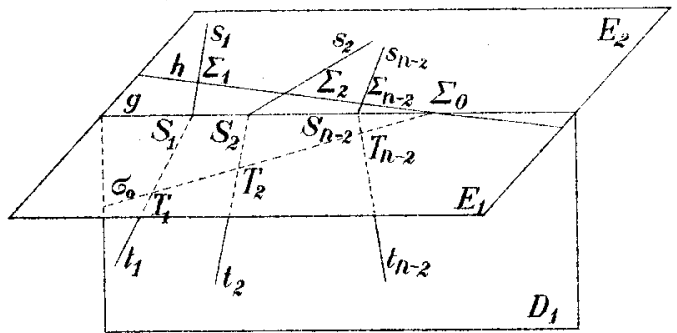

Fig. 6.

$s$ liegt. Eine solche Gerade $h$ läßt sich stets konstruieren; wir brauchen nur auf der Geraden $g$ den Punkt $\Sigma_{0}$ entweder im Stück $\infty S_{1}$ oder $S_{n-2} \infty$ zu wählen (in den Fig. 4, 6 ist der letztere Fall dargestellt) und durch $\Sigma_{0}$ eine Gerade so zu ziehen, daß der in $E_{2}$ gelegene Halbstrahl mit $\Sigma_{0} S_{1}$ einen genügend kleinen Winkel bildet. 
In analoger Weise legen wir in der Ebene $D$ eine von $g$ verschiedene Gerade $\sigma_{0}$ durch $\Sigma_{0}$ derart, daß der in $D_{1}$ liegende Halbstrahl von $\sigma_{0}$ die Geraden $t_{1}, t_{2}, \ldots t_{n-2}$ im Endlichen in lauter verschiedenen Punkten $T_{1}, T_{2}, \ldots T_{n-2}$ schneidet und daß auf jeder der Geraden $t_{i}(i=1,2, \ldots n-2)$ zwischen $S_{i}$ und $T_{i}$ kein Schnittpunkt von $t_{i}$ mit irgend einer Schnittlinie des auf S. 4 definierten Systems 5 liegt (vgl. Fig. 5 und 6).

Die Ebene $F$ legen wir nun durch die Geraden $h, \sigma_{o}$. Die Schnittigur der Ebene $F$ mit den $(n+1)$ Polyedern und ihren Begrenzungsflächen veranschaulicht Fig. 7.

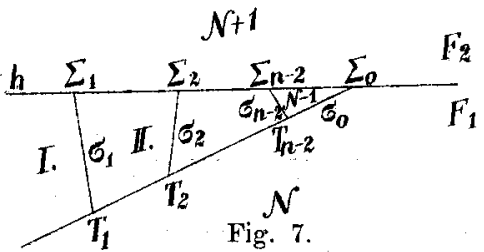

Daß Bedingung 3 a) für $F$ zutrifft, ist evident. Wenn wir nun mit $f_{1}, f_{2}$ die beiden durch $F$ gebildeten Halbräume bezeichnen, u. $\mathrm{zw}$. mit $f_{2}$ denjenigen, in welchem $S_{1}, S_{2}, \ldots S_{n-2}$ liegen, so genügt offenbar der Halbraum $f_{1}$ der in $3 \mathrm{~b}$ ) ron dem

Halbraum $e_{1}$ geforderten Bedingung.

Desgleichen ist $3 \mathrm{c}$ ) erfüllt, wobei $s_{1}, s_{2} \ldots s_{n-2} ; S_{1}, S_{2}, \ldots S_{n-2}$ durch $\sigma_{0}, \sigma_{1}, \ldots \sigma_{n-2} ; \Sigma_{0}, \Sigma_{1}, \ldots \Sigma_{n-2}$ zu ersetzen sind.

Die beiden Halbebenen, in die $F$ durch $h$ zerlegt wird, mögen mit $F_{1}, F_{2}$ bezeichnet werden, je nachdem sie im Halbraum $e_{1}$, bezw. $e_{2}$ liegen. Die Reste der $n$ ursprünglichen Polyeder, die bei der neuen Einteilung $\mathfrak{I}_{n+1}$ des Raumes übrig bleiben, erfüllen den Halbraum $e_{1}$; ihre Schnittfiguren mit $F$ liegen somit in $F_{1}$. Dieselben grenzen (s. Fig. 7) sämtlich an die Gerade $h$, so dab auch die Bedingung $3 \mathrm{~d}$ ) von der Ebene $F$ erfüllt wird.

Der Satz von S. 5 ist also bewiesen. $\mathrm{Da}$ man nun eine Teilung $\mathfrak{I}_{2}$ mit den verlangten Eigenschaften herstellen kann man braucht nur den Raum durch eine Ebene in zwei Halbräume zu teilen, - so ist damit auch gezeigt, daß man den Raum in beliebig viele konvexe Polyeder teilen kann, die ein System von Nachbargebieten darstellen. 